\title{
Free Vibrations of a Rotating Single-Walled Carbon Nanotube Embedded in an Elastic Medium Based on Nonlocal Elasticity Theory
}

\author{
H. NAHVi* AND M.E. Boroojeni \\ Islamic Azad University, Khomeinishahr Branch, Khomeinishahr, Isfahan 84175/119, Iran
}

\begin{abstract}
In this paper, a nonlocal beam model is developed and applied to investigate flapwise vibration characteristics of a rotating single-walled carbon nanotube on a Pasternak foundation. Differential quadrature method is used to solve equation of motion. The effects of small-scale factor, angular velocity and elastic foundation on the natural frequencies are examined and discussed. It is shown that small-scale effect plays an important role in the vibration response of a rotating nanotube.
\end{abstract}

DOI: 10.12693/APhysPolA.123.304

PACS: 62.25.-g, 62.25.De

\section{Introduction}

In recent years, carbon nanotubes $(\mathrm{CNT})$ have become the central point of researches in computational nanomechanics. Vibration analysis is of fundamental importance in the design of nanoelectromechanical systems. The state of the art on the vibration analyses of nanotubes can be found in a review work by Gibson et al. [1]. Beams supported on elastic foundations are usually incorporated in the design of air craft structures where CNTs are very important and useful in immense applications. Mechanical characteristics of CNTs embedded in polymer environment have been studied in [2] The effect of elastic foundation on the single-walled CNT (SWCNT) vibration has been studied by Wang et al. [3]. Mustapha and Zhong investigated vibrations of an axially loaded SWCNT embedded in a two-parameter elastic medium [4].

In this study, vibration characteristics of a rotating single-walled cantilever carbon nanotube embedded in a deformable surrounding medium are investigated. The SWCNT is modeled as a nonlocal Rayleigh beam of length $L$ and Pasternak-type foundation model is employed to simulate the interaction of SWCNT with surrounding elastic medium. The governing differential equation for SWCNT vibration is solved using differential quadrature (DQ) method. It has been shown that the fundamental frequency of a rotating SWCNT cantilever strongly depends on the nonlocal parameter, angular velocity and stiffness of the surrounding medium. The analyses further illustrates that the nonlocal parameter has different effects on different vibration modes.

\section{Governing equations}

According to Eringen's theory, the stress field at one point in an elastic continuum depends on the strains at

*corresponding author; e-mail: nahvi@iaukhsh.ac.ir all other points of the body. The basic equations for a homogeneous nonlocal elastic body are presented by Eringen [5].

The one-dimensional nonlocal relation for a general Rayleigh beam can be written as

$$
\sigma^{\mathrm{nl}}(x)-\left(e_{0} a\right)^{2} \frac{\partial^{2} \sigma_{x x}^{\mathrm{nl}}}{\partial x^{2}}=E \varepsilon(x),
$$

where $\sigma^{\text {nl }}(x)$ is the nonlocal stress, $e_{0} a$ is the nonlocal parameter, $E$ is the Young modulus and $\varepsilon(x)$ is the nonlocal strain [5].

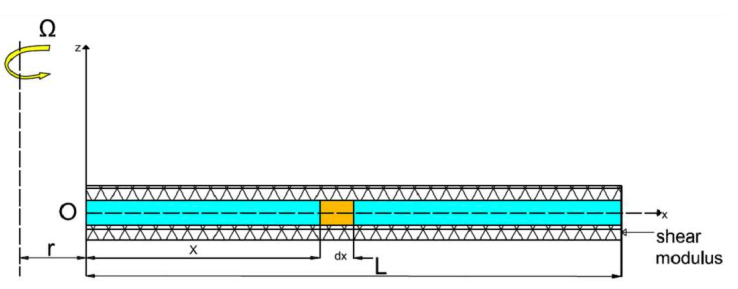

Fig. 1. Configuration of a rotating Euler-Bernoulli SWCNT on a Pasternak foundation.

Shown in Fig. 1 is a SWCNT nanocantilever of length $L$, which is fixed at point $O$ to a rigid hub that rotates at a constant rotational speed and rested on a Pasternak foundation. Based on the Eringen nonlocal theory, the Euler-Lagrange equation for the Rayleigh beam is obtained as [4]:

$$
\begin{aligned}
& \frac{\partial^{2} M_{x x}}{\partial x^{2}}+q-\frac{\partial}{\partial x}\left(N_{x x} \frac{\partial w}{\partial x}\right)-\rho A \frac{\partial^{2} w}{\partial t^{2}}+\rho I \frac{\partial^{4} w}{\partial t^{2} \partial x^{2}} \\
& \quad-K_{\mathrm{G}} \frac{\partial^{2} w}{\partial t^{2}}=0,
\end{aligned}
$$

where $M_{x x}, w, q, \rho A, \rho I, K_{\mathrm{G}}$, and $A$ are the resultant moment stress, transverse displacement, tension force, mass per unit length, rotary inertia, shear modulus parameter of the deformable medium and cross-sectional area, respectively. The centrifugal tension force due to the rotation of the cantilever, $N_{x x}$, at a distance $x$ from the origin (Fig. 1) is given by 


$$
N_{x x}=\int_{x}^{l} \rho A \Omega^{2}(r+x) \mathrm{d} x,
$$

where $\Omega$ is the angular velocity of the nanotube cantilever and $r$ is the hub radius.

Using Eqs. (2) and (3), the governing differential equation for the flapwise bending vibration of the rotating nanotube can be obtained as

$$
\begin{aligned}
& E I \frac{\partial^{4} w}{\partial x^{4}}+\rho A \omega^{2} w+\left(e_{0} a\right)^{2} \rho A \omega^{2} \frac{\partial^{2} w}{\partial x^{2}} \\
& \quad-\left(e_{0} a\right)^{2} \rho I \omega^{2} \frac{\partial^{4} w}{\partial x^{4}}+\rho I \omega^{2} \frac{\partial^{2} w}{\partial x^{2}}-\rho A \Omega^{2} \\
& \quad \times\left\{(-r-x) \frac{\partial w}{\partial x}+r(L-x)+0.5\left(L^{2}-x^{2}\right) \frac{\partial^{2} w}{\partial x^{2}}\right] \\
& +\left(e_{0} a\right)^{2} \rho A \Omega^{2}\left[-3 \frac{\partial^{2} w}{\partial x^{2}}+3(-r-x) \frac{\partial^{3} w}{\partial x^{3}}\right. \\
& \left.+\left[r(L-x)+0.5\left(L^{2}-x^{2}\right)\right] \frac{\partial^{4} w}{\partial x^{4}}\right\} \\
& \quad+\left(e_{0} a\right)^{2} k_{\mathrm{G}} \frac{\partial^{6} w}{\partial x^{6}}-k_{\mathrm{G}} \frac{\partial^{2} w}{\partial x^{2}}=0 .
\end{aligned}
$$

For a cantilever beam, the four boundary conditions at the two ends are given by

$$
\begin{aligned}
& w=\frac{\partial w}{\partial x}=0 \quad \text { at } \quad x=0 \\
& \text { and } \frac{\partial^{2} w}{\partial x^{2}}=\frac{\partial^{3} w}{\partial x^{3}}=0 \quad \text { at } \quad x=l .
\end{aligned}
$$

\section{Numerical results and discussion}

A code is written in Matlab to solve Eq. (4) and perform DQ method. Fifteen grid points are used for convergence of the DQ approach [6]. At first, the effect of surrounding elastic foundation is neglected, i.e. $K_{\mathrm{G}}=0$. Figure 2 depicts the variations of 1 st frequency deviation percent of the rotating SWCNT in terms of the non-dimensional angular velocity $\gamma\left(\gamma^{2}=\frac{\rho A \Omega^{2} L^{4}}{E I}\right)$, for both local and nonlocal elastic models. Frequency deviation percent is defined as:

Frequency deviation percent

$$
=\frac{100 \times(\text { frequency }(\text { local })-\text { frequncy }(\text { nonlocal }))}{\text { frequency }(\text { local })} \text {. }
$$

From Fig. 2 it can be seen that for all values of the nonlocal parameter, as the non-dimensional angular velocity increases, the frequency deviation percent increases as well. This phenomenon occurs in both local and nonlocal elastic models. The increase of the frequency deviation percent with angular velocity is attributed to the stiffening effect of the centrifugal force which is proportional to the square of the angular velocity.

The effect of small-scale on the 2nd mode of the rotating SWCNT is shown in Fig. 3. Unlike the 1st mode, frequency deviation percent values of the local model are

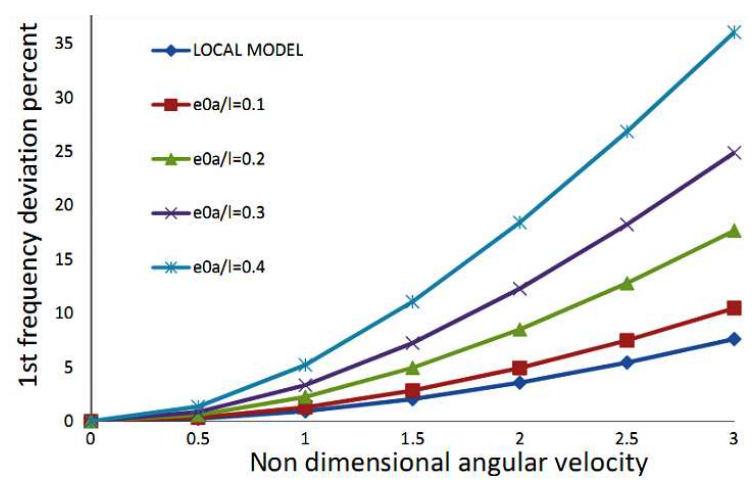

Fig. 2. Variations of 1st frequency deviation percent versus non-dimensional angular velocity of rotating SWCNT, $K_{\mathrm{G}}=0$.

more than that of the nonlocal models. It is also observed that for a certain angular velocity, with increase of the nonlocal coefficient, the frequency deviation percent decreases.

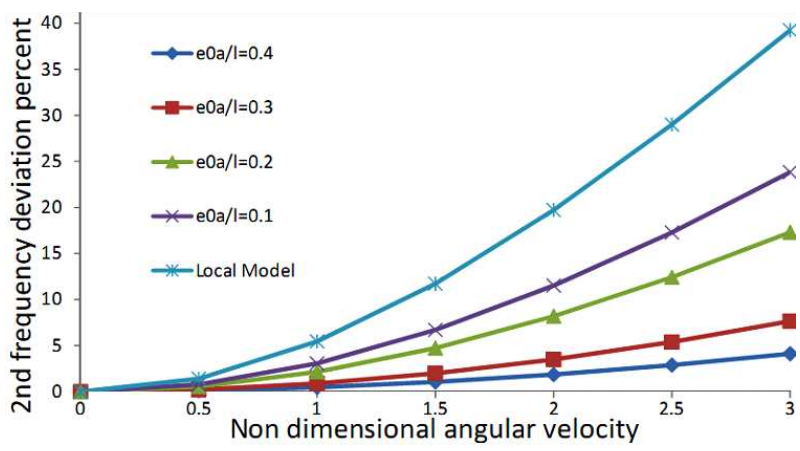

Fig. 3. Variations of 2 nd frequency deviation percent versus non-dimensional angular velocity of rotating SWCNT, $K_{\mathrm{G}}=0$.

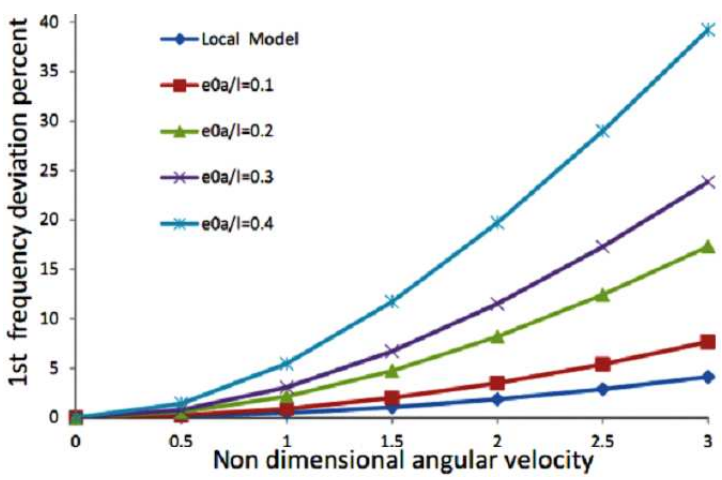

Fig. 4. Variations of 1st frequency deviation percent of SWCNT on elastic foundation versus non-dimensional angular velocity, $K_{\mathrm{G}}=2$.

The variations of the fundamental frequency deviation percent of SWCNT versus non-dimensional angular ve- 


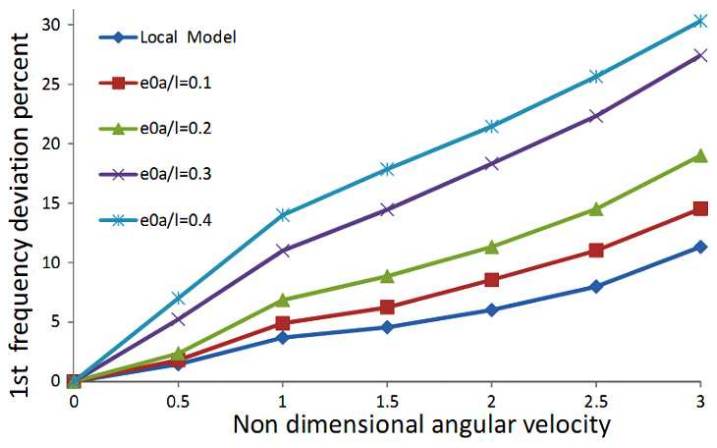

Fig. 5. As in Fig. 4, but for $K_{\mathrm{G}}=10$.

locity for the Pasternak-type elastic medium for two stiffnesses $K_{\mathrm{G}}=2$ and 10 are respectively shown in Figs. 4 and 5 , respectively. From these figures it can be seen that in both local and nonlocal cases, by increasing the angular velocity, fundamental frequency deviation percent increases. Moreover, as the Pasternak coefficient increases, the rate of the increase of the frequency deviation percent decreases.

\section{Conclusions}

Nonlocal elasticity beam model is applied to study the vibration response of rotational SWCNT. The DQ method is utilized to solve equation of motion. It is observed that for the first mode of vibration, the frequencies obtained with nonlocal model are higher than those computed with local model; but for the second mode it is adverse. By increasing the angular velocity, the difference between the local and nonlocal frequencies increases. For the Pasternak elastic medium, it is shown that fundamental frequency deviation percent increases for both nonlocal and local cases and with the same angular velocity, its values are less than those for the model without elastic medium.

\section{References}

[1] R.F. Gibson, E.O. Ayoriend, Y.F. Wen, Composit. Sci. Technol. 67, 1 (2007)

[2] A.R. Ranjbartoreh, A. Ghorbanpour, B. Soltani, Physica E, Low-Dimens. System Nanostruct. 39, 230 (2007)

[3] L. Wang, Q. Ni, M. Li, Q. Qian, Physica E, Low-Dimens. System Nanostruct. 40, 3179 (2008)

[4] K.B. Mustapha, Z.W. Zhong, Comput. Mater. Sci. 50, $742(2010)$

[5] A.C. Eringen, Nonlocal Continuum Field Theories, Springer-Verlag, New York 1970.

[6] C. Shu, Differential Quadrature and Its Application in Engineering, Springer-Verlag, London 2000. 